\title{
PREVALENCE OF BEHAVIORAL RISK FACTORS OF NON COMMUNICABLE DISEASES AMONG ADOLESCENTS OF HIGHER SECONDARY SCHOOLS OF LALITPUR DISTRICT IN NEPAL
}

\author{
Soni Shrestha ${ }^{1}$, Radha Kumari Paudel ${ }^{2}$ and Nisha Shrestha ${ }^{3}$
}

1Soni Shrestha, Phd Scholar, Mewar University, Rajasthan ,India, sonishrestha2003@yahoo.com

${ }^{2}$ Radha Paudel, , Phd Scholar, Mewar University, Rajasthan, India

${ }^{3}$ Nisha Shrestha, Undergraduate Student, Manmohan Memorial Institute of Health Science

\begin{abstract}
Non Communicable Diseases are the biggest cause of death worldwide. There are eight major risk factors, out of which four of them are behavioral risk factors (tobacco use, harmful alcohol consumption, unhealthy diets and physical inactivity) and four of them are biological risk factors. The general objective of this study is to assess prevalence of behavioral risk factors of noncommunicable diseases among adolescents. The study was conducted on students of higher secondary schools of Lalitpur. A descriptive cross-sectional study was conducted with the sample size 396. Structured questionnaire developed by STEPS SURVEY Nepal 2013 was used for data collection. Data management, analysis and interpretation were done using SPSS 20 version by calculating frequency and percentage. The prevalence of current tobacco (smoking) consumption was $17.2 \%$ and the prevalence of smokeless tobacco consumption was 8.1\%. Current alcohol consumption was $14.4 \%$. The prevalence of inadequate vigorous intensity activity was $58.3 \%$ and moderate intensity activity was $50.5 \%$. The prevalence of fruit and vegetables consumption was $21.2 \%$ and $60.4 \%$ respectively. On the basis of findings of study it is concluded that use of tobacco products and alcohol was higher in male than female. All of the students consumed fruits and vegetables at least once a day but none of adolescents follow the consumption of fruits and vegetables as recommended by WHO. The adolescents were active as they involved in both vigorous and moderate intensity activity. The private schools students were less involved in physical activity as compared to public schools.
\end{abstract}

\section{KEYWORDS}

Adolescents, Behavioral risk factors, Non-communicable diseases

\section{INTRODUCTION}

Non communicable disease can be defined as disease or conditions that occur in, or are known to affect, individuals over extensive period of time and for which there are no known causative agents that are transmitted from one affected individual to another. They are of long 
duration and generally slow progression. The 4 main types of non-communicable diseases are cardiovascular diseases (like heart attacks and stroke), cancers, chronic respiratory diseases (such as chronic obstructed pulmonary disease and asthma) and diabetes (WHO, 2014).There are eight major risk factors ,out of which four of them are behavioral risk factors and four of them are biological risk factors. The behavioral risk factors include tobacco use, harmful alcohol consumption, unhealthy diets and physical inactivity. Non-communicable diseases (NCDs) are the resultant morbidity and mortality due to them is ever increasing (Galhotra, 2009). Cardiovascular and other chronic diseases are becoming the major causes of morbidity and mortality in most of the third world countries, including Nepal (Adhikari, 2012). Deaths from NCDs are projected to rise from 4 million to 8 million a year in India. Young and adolescents are susceptible to unhealthy lifestyle and have been shown to have various risk factors that may predispose them to development of NCDs. The World Health Organization has already warned of increasing NCDs among adolescents as a major public health problem (R.Sogarwal, 2014).

A life course approach to preventive efforts addressing NCDs and their risk factors and behaviors will improve child and adolescent health but also decrease lifetime health care costs (Catalano RF F. A., 2012). Alcoholism, tobacco smoking, unhealthy diet are prevalent among adolescents and almost $80 \%$ of deaths due to NCDs can be prevented by eliminating those risk factors. Nevertheless, those risk behaviors are initiated usually in the adolescent's age groups which are continued to adult. Therefore, this group is important target for primordial prevention. NCDs affect large numbers of people under the age of 60 and exact a huge toll on health, the economy, and human potential. The prevalence of NCDs is related to unhealthy behaviors and practices typically initiated in adolescence. Given that one in four people in Latin America and Caribbean is between the ages of 10 and 24, these unhealthy behaviors among young people will have a direct effect on their risk of developing NCDs later in life. Building a healthier future depends on effective interventions during this critical window of opportunity. The four main NCDs are driven by four modifiable risk behaviors: tobacco use, excessive use of alcohol, unhealthy diet, and insufficient physical activity (WHO, 2011). These behaviors can lead to overweight and obesity, high blood pressure, and high cholesterol-all directly related to NCDs. (The World Health Organization defines adults as overweight when their body mass index (BMI) is greater than or equal to 25 and obesity as a BMI greater than or equal to 30 (R.Sogarwal, 2014). Adolescents and youth are a tremendous resource that is overlooked in the fight against NCDs, yet they are a natural partner for preventing NCDs. The World Health Organization estimates that 70 percent of premature deaths in adults are the result of behaviors begun during adolescence and youth. Research indicates that behaviors associated with two of the key risk factors for NCDstobacco and alcohol use - are likely to start or become established during adolescence. Other risk factors related to poor diet and insufficient physical activity may begin during childhood, but adolescence is an opportunity to reinforce the benefits of positive behaviors through appropriate messages and programs. Experts estimate that the projected burden of NCDs could be cut in half or more by focusing on health promotion and disease prevention (The World Bank.The Growing Danger of Non-Communicable Disease). As a researcher preventive strategies can be formulated 
regarding NCDs risk factors on schools as well as policies can be formulated on the school basis regarding prevention of NCDs for behavioral skill training, for promotion of sports and recreational activities and for tobacco free settings.

\section{MATERIALS AND METHODS}

A quantitative descriptive cross sectional study was conducted among higher secondary schools students of Lalitpur districts. Late adolescent's period i.e. 15-19 years of students were assessed. Probability Stratified sampling was used to choose higher secondary schools. Out of 49 schools, nine schools were selected by doing random sampling and sample size was 396 with 10 $\%$ non-response rate. Structured questionnaire was developed by STEPS SURVEY Nepal 2013 which consists of three Steps for measuring NCD risk factors. Step I includes socio- demographic and behavioral information which included tobacco use, harmful alcohol consumption, unhealthy dietary pattern. The pretesting was done among $10 \%$ of adolescents of higher secondary schools of Kathmandu.

The data was collected during three weeks period of month August, 2016. Formal permission was obtained from different higher secondary schools. Verbal consent with the respective school's principal and the respondents was taken before data collection Ethical approval was taken from Institute Review Board of MMIHS.

\section{RESULTS}

\section{Demographic characteristics of respondents}

Out of 396 respondent's majority $50.5 \%$ of respondents were female and $49.5 \%$ of respondents were male. Majority $65.9 \%$ of respondents were of age 15-17 years. The respondents from private schools $56.1 \%$ were much greater in number than public schools $43.9 \%$. The majority $69.9 \%$ of respondents were from Hindu religion. The majority $64.6 \%$ of respondent's family monthly income was Rs 10000-30000 (Table 1).

Table 1. Demographic characteristic of the respondents.

\begin{tabular}{ll}
\hline Characteristics & Percentage (\%) \\
\hline Age & 65.9 \\
15-17years & 34.1 \\
$>17$ years & \\
Sex & 49.5 \\
Male & 50.5 \\
Female & \\
Type of school & \\
Private & 43.9 \\
Public & 56.1 \\
\end{tabular}




\begin{tabular}{ll} 
Religion & \\
Hindu & \\
Buddhist & 69.9 \\
Muslim & 15.9 \\
Kirat & 0.8 \\
& 3 \\
Income Rs & \\
10000-30000 & 64.9 \\
31000-60000 & 29.5 \\
60000-90000 & 4.5 \\
Above 90000 & 1.3 \\
& \\
\hline
\end{tabular}

Table 2 shows the behavioral risk factors of non-communicable diseases. The prevalence of current tobacco (smoking) consumption was $17.2 \%$ and the prevalence of smokeless tobacco consumption was $8.1 \%$. The prevalence of current alcohol consumption was $14.4 \%$ ). The prevalence of inadequate vigorous intensity activity was $58.3 \%$ and moderate intensity activity was $50.5 \%$ ). The prevalence of fruit consumption and vegetables consumption was $21.2 \%$ ) and $60.4 \%$ respectively.

Table 2. Prevalence of behavioral risk factors.

\begin{tabular}{ll}
\hline Behavioral Risk Factors & Percentage (\%) \\
\hline Current tobacco consumption & 17.2 \\
Current alcohol consumption & 14.4 \\
Inadequate moderate & 50.5 \\
intensity exercise & 58.3 \\
Inadequate vigorous intensity & 21.2 \\
exercise & \\
Fruit Consumption & 60.4 \\
Vegetable Consumption & \\
\end{tabular}

\section{DISCUSSION}

Demographic findings revealed that $50.5 \%$ of respondents were female and most of respondents $65.9 \%$ were $15-17$ years of age group. Majority $71.7 \%$ of respondents were from grade 11. The respondents from private schools $56.1 \%$ were much greater in number than public schools $43.9 \%$. As per the classification of ethnicity, majority $34.6 \%$ of respondents were from janajati. 
The majority $69.9 \%$ of respondents were from Hindu religion. The majority $64.6 \%$ of respondent's family monthly income was Rs $10000-30000$. Majority $37.9 \%(\mathrm{n}=150)$ of respondent's mothers were illiterate. The majority $32.1 \%$ of respondent's fathers were educated to secondary level. Majority of $23.5 \%$ of respondent's father's occupation was agriculture and majority $41.2 \%$ of respondent's mother's occupation was house-maker. In this study $17.2 \%$ of respondents were currently using tobacco products in any form which is consistent to study conducted by Andey et al. (2002) on school children of grade 8, 9 and 10 in central region of Nepal that has reported over all $16.3 \%$ of respondents ever used tobacco product in any form and the rate among boys was significantly higher than that among girls. A study conducted by (Karki KB, 2008) revealed that the overall smoking prevalence in Nepal for the population aged fifteen or more is $37.4 \%$ which is significantly higher than present study. The difference in finding of the study may be due to small sample size of this study. Similar study conducted by (Karki, 2015) revealed that $7.1 \%$ of respondents has currently consumed smokeless tobacco products which is similar to present study that is $8.1 \%$ The present study shows that $6.1 \%$ of respondents were past users of smokeless tobacco products which is higher than study conducted by Katel (2015). In this study, the mean age of initiation of smoking was 15.18 years which is consistent to the findings of study conducted by (Karki, 2015) where mean age of initiation of smoking was 17.38 years and 16.42 years respectively.

The present study revealed that $14.4 \%$ of respondents were currently consuming alcohol which is similar to (Krishna Kumar Aryal, 2013) that is $17 \%$.The study conducted by Jain et.al (2012)alcohol consumption was done by $4.6 \%$ students which is inconsistent to findings of present study. The inconsistent may be due to difference in settings. Similar study conducted among adolescents in Nepal has found $26.8 \%$ male and 18.2\% female respondents were consuming alcohol that revealed male was significantly consuming alcohol higher than that female (Adhikari, 2012).

In this study respondents consumed fruits four days per week and vegetables for 6 days per week which is consistent to the study conducted by (Karki, 2015) i.e. fruit consumption was 3.15 days per week and vegetables consumption was 6.42 days. The findings of (Krishna Kumar Aryal, 2013) shows the mean number of days of vegetables consumed was 4.8 days per week which is lower than present study. The dissimilarity may be due to small sample size of present study. In this study, all respondents consumed fruits and vegetables daily of which $21.2 \%$ consumed fruits daily and $60.4 \%$ consumed vegetables daily which are consistent to study 43 conducted by (Animesh, 2012). In response to salt consumption, in this study $8.1 \%$ of respondent's often added salt before eating, $13.6 \%$ of students often eat processed food high in salt.

This finding was supported by study done in Nepal which showed around $5.7 \%$ of the respondent always or often added salt before eating, $14.1 \%$ respondents often consumed processed food containing high amounts of salt (Karki, 2015). In this study $41.7 \%$ of respondents performed vigorous intensity activity and $49.5 \%$ of respondents performed moderate intensity activity which 
is contrast to the findings of (Animesh, 2012) that is $82.6 \%$ vigorous intensity activity and $98.5 \%$ moderate intensity activity. The inconsistent may be due to lack of knowledge.

\section{CONCLUSIONS}

The use of tobacco products and alcohol was higher in male than female. The uses of tobacco products are higher among students of public schools than in private schools. The adolescents were active as they involved in both vigorous and moderate intensity activity

The private schools students were less involved in physical activity as compared to public schools. All of the students consumed fruits and vegetables at least once a day but none of adolescents follow the consumption of fruits and vegetables as recommended by WHO. Awareness programs regarding harmful effects of alcohol and tobacco use should be focused on male students and emphasized should be given in public schools.

\section{REFERENCES}

Adhikari, K., \& Adak, M.R. (2012). Behavioural risk factors of non-communicable diseases. Journal of Institute of Medicine , 34 (3), 39-43.

Animesh, J., Dhanawat, J., Kotian, M.S, \& Angeline, R. (2012). Assessment of risk factors of noncommunicable diseases among high school student. 1 (4), 249-254.

Catalano, R.F., Fagan, A. A., Gavin, L.E., Greenberg, M.T., Irwin, C.E., Ross, D.A., \& Shek, D.T.L. (2012). Worldwide application of prevention science in adolescent health. Lancet, 379, 1653-1664.

Galhotra, A., Abrol, A., Goel, N., \& Gupta, S. (2009). Life style related Risk Factors for Cardiovascular Diseasea in Indian Adolscents. The Internet Journal of Health , 9 (2), 87-89.

Karki, K.B. (2008). WHO STEPS Surveillance: Non Communicable Diseases Risk Factors Survey. Society for Local Integrated Development Nepal (SOLID Nepal) and WHO, Ka.

Karki, B. K. (2015). Behavioral risk factors of non communicable diseases among adult women in Kathmandu. Master's Thesis.

Aryal, K.K., \& Neupane, S.(2013). Non Communicable Disease Risk Factors. STEPS Survillence in nepal.

Sogarwal, R., Bachani, B., Kumar, B., \& Gupta, S. (2014). Risk factors of Non-Communicable Diseseses among Higher Secondary School Students in Selected Districts of India. American Journal of Public Health Research, 2 (1), 16-20.

Baldwin, W., Kaneda, T., Amato, L., \& Nolan, L. (2013, June). Non Communical diseases and youth: A critical window of opperrtunity for latin American and the caribeian.

WHO. (2011). Global status report on non-communicable disease . Geneva.

WHO. (2014). Global status report on non communicable diseases . Geneva . 
ISSN: 2362-1303 (Paper) | elSSN: 2362-1311(Online)

JOURNAL OF ADVANCED ACADEMIC RESEARCH (JAAR)

April 2017 